\title{
Avaliação da produção de drogas vegetais, derivados vegetais e fitoterápicos do Laboratório de Manipulação Farmacêutica para fornecimento ao Programa de Fitoterapia e Homeopatia da Secretaria Municipal da Saúde de Ribeirão Preto - SP
}

\author{
Júlio Cézar Borella ${ }^{\mathrm{a}, \mathrm{b}, *}$ e Juliana Coelho Lopes Teixeira ${ }^{\mathrm{c}}$
}

${ }^{\text {a}}$ Laboratório de Manipulação Farmacêutica da Secretaria da Saúde de Ribeirão Preto, Riberão Preto, SP, Brasil

${ }^{\mathrm{b}}$ Curso de Ciências Farmacêuticas, Universidade de Ribeirão Preto (UNAERP), Ribeirão Preto, SP, Brasil

${ }^{c}$ Laboratório de Manipulação Farmacêutica da Secretaria da Saúde de Ribeirão Preto, Ribeirão Preto, SP, Brasil

Histórico do Artigo

Recebido em:

02/11/2017

Aceito em:

08/02/2018

Palavras-chave:

Programa Nacional de

Saúde;

fitoterápicos;

produção de

medicamentos.

Key words:

National Health

Programs;

phytotherapeutic drugs; production of products.

\section{RESUMO}

O Programa de Fitoterapia e Homeopatia da Secretaria da Saúde de Ribeirão Preto foi um dos primeiros a atuar no estado de São Paulo, a partir de 1992. A demanda de produtos à base de plantas medicinais para este programa foi suprida pelo trabalho do Laboratório de Manipulação Farmacêutica. A partir dos dados relativos à produção deste setor, nos anos de atuação do programa (1994 até 2017), foi possível usar os resultados como parâmetro avaliativo da atuação deste programa no município de Ribeirão Preto - SP. Observou-se a existência de fases na atuação do programa, sendo a primeira (1994 a 2002) caracterizada pela diversidade de preparações (21 tipos) e um clínico com direito à prescrição. Nessa fase, houve a produção de 4.318 unidades medicamentosas, com maior demanda para Calendula officinalis pomada $10 \%$ $(17,07 \%)$, Maytenus ilicifolia folhas (9,82\%), Passiflora alata tintura $20 \%(7,43 \%)$ e Allium sativum cápsulas $500 \mathrm{mg}(7,27 \%)$. Outra fase (2003 a 2015) teve como características a drástica diminuição da diversidade de preparações [2 tipos - Calendula officinalis pomada $10 \%$ $(95,11 \%)$ e Stryphnodendron adstringens creme a $10 \%(4,89 \%)]$ e o aumento do número de unidades produzidas (31.311), pela possibilidade de indicação de uso ter sido ampliada para integrantes de equipes multiprofissionais da rede de saúde do município. Nos anos de $2016 \mathrm{e}$ 2017, o Laboratório de Manipulação Farmacêutica passou por séria crise produtiva, que estava associada à crise econômica e política, com consequente restrição na produção de itens para uso no programa.

Evaluation of the production of vegetal drugs, extracts and phytomedicines of the Pharmaceutical Manipulation Laboratory for supply to the Phytotherapy and Homeopathy Program of the Municipal Health Department of Ribeirão Preto - SP

\section{ABSTRACT}

The Phytotherapy and Homeopathy Program of the Health Department of Ribeirão Preto was one of the first to operate in the state of São Paulo, since 1992. The demand for herbal products for this program was supplied by the Pharmaceutical Manipulation Laboratory. From the data on the production of this sector, in the program's years of operation (1994 to 2017), it was possible to use the results as an evaluation parameter for the performance of this program in Ribeirão Preto - SP. It was observed existence of phases in the program's performance. The first one (1994 to 2002) characterized by the diversity of preparations (21 types) and one medical with right of prescription. At this stage there were 4,318 drug units, with a higher demand for Calendula officinalis ointment at 10\% (17.07\%), Maytenus ilicifolia leaves 100g $(9.82 \%)$, Passiflora alata tincture at $20 \%(7,43 \%)$ and Allium sativum capsules 500mg (7,27\%). Another phase (2003 to 2015) had a drastic decrease in the diversity of preparations [2 types - Calendula officinalis ointment at 10\% (95,11\%) and Stryphnodendron adstringens cream at $10 \%(4,89 \%)$ ] and the increase in the number of units produced ( 31,311 units), by the possibility of indication of use has been extended to members of the multiprofessional teams of the health network of the municipality. In 2016 and 2017, the Pharmaceutical Manipulation Laboratory went through a serious productive crisis that was associated with the economic and political crisis, with a consequent restriction on the production of items for use in the program.

\footnotetext{
${ }^{*}$ Autor correspondente: jborella@unaerp.br (J. C. Borella)
} 


\section{Introdução}

Foi a partir dos anos 1980, após a Conferência Internacional sobre Cuidados Primários de Saúde (Declaração de Alma Ata), que se iniciou, em algumas sociedades ao redor do globo, o retorno à utilização de terapêuticas "mais saudáveis" para cura de suas enfermidades (1). Essa atitude pode ser parcialmente explicada pelo sentimento negativo demonstrado por essas populações em relação às terapêuticas alicerçadas no uso de fármacos sintéticos, majoritariamente utilizadas até então, com grande incidência de efeitos adversos apresentados por esses medicamentos (2).

Entre as terapêuticas que voltaram a ser solicitadas pela população, a fitoterapia teve importância destacada. Terapêutica ancestral para enfrentar as enfermidades que sempre afligiram os seres humanos e que se caracteriza pelo uso de espécies vegetais "in natura" ou na forma de drogas vegetais, ou de seus extratos (derivados vegetais), ou, ainda, de uma forma mais elaborada, através dos fitoterápicos (medicamentos com forma farmacêutica definida, tendo como princípios ativos o complexo de substâncias que está presente nos derivados vegetais ou nas próprias plantas) (3). Nota-se que, mesmo na atualidade, este movimento mundial de valorização e uso de plantas medicinais e fitoterápicos ainda continua evidente (4).

No Brasil, programas governamentais e legislações para o desenvolvimento e uso da fitoterapia no setor público foram efetivados a partir desses acontecimentos. Desse modo, podem ser citados como exemplos, em 1982, o Programa de Pesquisa de Plantas Medicinais da Central de Medicamentos, com finalidade de estudar cientificamente, dando ênfase para ação farmacológica de espécies medicinais mais utilizadas no Brasil (5) e Resolução CIPLAN-8 (1988), que instituiu e regulamentou a fitoterapia no Sistema Público de Saúde (6).

Nos anos 2000, novas políticas públicas voltadas para este tema (Política Nacional de Práticas Integrativas e Complementares e Política Nacional de Plantas Medicinais e Fitoterápicos) $(7,8)$ foram efetivadas no sentido de dar novo avanço e ampliar acesso desta terapêutica no SUS. A partir desse momento, houve maior adesão dos estados e municípios para o desenvolvimento de projetos destinados a essa finalidade, com intensão de efetivar as cadeias produtivas locais necessárias para produção desse tipo de medicamento (9).

Ribeirão Preto, município do interior paulista, com cerca de 680 mil habitantes, possui extensa rede pública de saúde com 26 unidades básicas de saúde (UBS), 15 unidades de saúde da família (USF), 3 unidades básicas e especializadas, 2 unidades básicas e distritais de saúde (UBDS), 2 unidades distritais e especializadas, 1 pronto atendimento (UPA) e 11 unidades especializadas (10). Foi o primeiro município paulista a estabelecer um programa institucionalizado de Fitoterapia, Homeopatia e Acupuntura junto à Secretaria da Saúde. Implantado em 1992, tinha como estruturação inicial o atendimento ambulatorial de Fitoterapia no Núcleo de Gestão Assistencial, com um clínico para essa especialidade, além de atuar fortemente em ações com a comunidade (implantação de "Farmácias Vivas", Rodas de Conversa com temas relacionados ao uso e preservação de espécies medicinais, entre outros.) $(11,12)$.

Implantado a partir dos anos 1990, o Laboratório de Manipulação Farmacêutica da Secretaria Municipal da Saúde se responsabilizou pela produção de drogas vegetais, derivados vegetais e fitoterápicos que o Programa de Fitoterapia e Homeopatia demandava e até os dias atuais essa realidade se mantém. Inicialmente, o trabalho foi desenvolvido somente pelo farmacêutico responsável. Após 2008, um auxiliar de farmacêutico foi disponibilizado para ajudar nas atividades do Laboratório de Manipulação. 
O processo de produção desses produtos sempre foi alicerçado no controle de qualidade dos insumos e produtos acabados. No decorrer desse período, sempre houve intenção de adequação às novas legislações que entraram em vigor (RDC 67/2007, RDC 18/2013). Houve, também, por parte dessa unidade, o comprometimento em relação ao desenvolvimento de novas técnicas de produção e metodologias analíticas através do desenvolvimento de projetos de pesquisa em associação com a Universidade de Ribeirão Preto (UNAERP) e, eventualmente, com apoio financeiro da Fundação de Amparo à Pesquisa do Estado de São Paulo (FAPESP) (13). Foi através dessas oportunidades de parcerias que houve condições para o desenvolvimento de ações relacionadas com o controle de qualidade dos insumos e produtos preparados.

Em 2006, usando uma espécie constante na Relação Nacional de Plantas Medicinais de Interesse ao SUS (RENISUS), o projeto intitulado "Padronização do processo de produção de fitoterápico para uso tópico de inflorescências de Calendula officinalis L. (Asteraceae) utilizado na rede SUS de Ribeirão Preto" foi executado nesses moldes e, como resultado, pode-se destacar o desenvolvimento de métodos de controle de qualidade para esse produto, aumento da quantidade produzida, além da publicação de sete artigos científicos sobre o tema (14-20), sendo que um deles (14) foi utilizado como referência na monografia para a calêndula, na $1^{a}$. edição do Memento Fitoterápico da Farmacopeia Brasileira (21).

Os medicamentos homeopáticos dispensados pelo programa, de início, foram adquiridos diretamente de farmácias de manipulação do município e o fluxo para dispensação não passava pelo Laboratório de Manipulação Farmacêutica.

No entanto, neste espaço temporal (1994 até 2017), vários momentos de avanço e retrocesso foram vividos pelo Programa de Fitoterapia e Homeopatia, conforme as circunstâncias econômicas e políticas local, estadual e federal.

Dessa forma, o presente trabalho objetiva a avaliação da produção do Laboratório de Manipulação Farmacêutica destinada ao Programa de Fitoterapia e Homeopatia, no período entre 1994 até 2017, na tentativa de usar esses resultados como parâmetro avaliativo da atuação desse programa no município de Ribeirão Preto - SP.

\section{Material e métodos}

O estudo, de caráter quantitativo e descritivo, foi realizado no Laboratório de Manipulação Farmacêutica da Secretaria da Saúde de Ribeirão Preto, setor responsável pela produção das drogas vegetais, derivados vegetais e fitoterápicos que se destinam ao Programa de Fitoterapia e Homeopatia da Secretaria da Saúde de Ribeirão Preto.

Para a coleta dos dados, foram utilizadas, de 1994 a 2005, informações sobre o histórico de produção do Laboratório de Manipulação Farmacêutica, detalhado na forma de notas de produção escrituradas nos livros de produção.

De 2006 a 2017, as informações foram coletadas a partir das fichas de produção de medicamentos, procedimento implantado a partir de 2006 para dar rastreabilidade às formulações produzidas.

Avaliaram-se, para cada nota de produção ou ficha de produção, o tipo de produto manipulado, apresentação e quantidade produzida. A análise quantitativa dos dados consistiu da distribuição das frequências absolutas de produção das formulações nos anos analisados. As informações foram compiladas e inseridas em planilhas de dados (Microsoft Excel 2010). 


\section{Resultados e discussão}

A tabulação dos dados obtidos a partir das notas de produção e das fichas de produção resultou num quadro da produção dos produtos à base de plantas medicinais elaborados no Laboratório de Manipulação Farmacêutica e que foram destinados ao Programa de Fitoterapia e Homeopatia da Secretaria da Saúde de Ribeirão Preto, no período de 1994 a 2017. Esses dados estão apresentados nas Tabelas 1 e 2.

Tabela 1: Número de unidades farmacêuticas contendo espécies medicinais produzidas no Laboratório de Manipulação Farmacêutica (1994-2002)

\begin{tabular}{|c|c|c|c|c|c|c|c|c|c|c|}
\hline Produto manipulado/Ano & 94 & 95 & 96 & 97 & 98 & 99 & 00 & 01 & 02 & Total \\
\hline $\begin{array}{l}\text { Ageratum conyzoides } \\
\text { partes aéreas } 100 \mathrm{~g}\end{array}$ & 30 & 50 & 27 & 25 & 35 & 7 & 0 & 0 & 0 & 174 \\
\hline $\begin{array}{l}\text { Allium sativum } \\
500 \mathrm{mg} \text { caps. c/ } 30 \text { unid. }\end{array}$ & 0 & 36 & 59 & 65 & 118 & 0 & 13 & 23 & 0 & 314 \\
\hline $\begin{array}{l}\text { Baccharis trimera } \\
\text { partes aéreas } 100 \mathrm{~g}\end{array}$ & 0 & 30 & 16 & 20 & 8 & 10 & 6 & 15 & 0 & 105 \\
\hline $\begin{array}{l}\text { Bauhinia forficata } \\
\text { tintura } 20 \% 100 \mathrm{~mL}\end{array}$ & 0 & 51 & 26 & 17 & 37 & 0 & 15 & 25 & 0 & 171 \\
\hline $\begin{array}{l}\text { Calendula officinalis } \\
\text { pomada } 10 \% 200 \mathrm{~g}\end{array}$ & 36 & 70 & 151 & 149 & 138 & 47 & 25 & 46 & 75 & 737 \\
\hline $\begin{array}{l}\text { Calendula officinalis } \\
\text { tintura } 20 \% 100 \mathrm{~mL}\end{array}$ & 10 & 20 & 10 & 20 & 26 & 12 & 15 & 10 & 0 & 123 \\
\hline $\begin{array}{l}\text { Cecropia glaziouvii } \\
\text { folhas } 100 \mathrm{~g}\end{array}$ & 20 & 15 & 0 & 0 & 0 & 0 & 0 & 0 & 0 & 35 \\
\hline $\begin{array}{l}\text { Cecropia glaziouvii } \\
\text { tintura } 20 \% 100 \mathrm{~mL}\end{array}$ & 19 & 45 & 37 & 63 & 62 & 20 & 0 & 20 & 10 & 276 \\
\hline $\begin{array}{l}\text { Cymbopogon citratus } \\
\text { folhas } 100 \mathrm{~g} *\end{array}$ & 30 & 0 & 45 & 0 & 0 & 0 & 0 & 0 & 0 & 75 \\
\hline $\begin{array}{l}\text { Matricaria recutita } \\
\text { inflores. } 50 \mathrm{~g}\end{array}$ & 30 & 41 & 29 & 81 & 63 & 16 & 0 & 30 & 0 & 290 \\
\hline $\begin{array}{l}\text { Maytenus ilicifolia } \\
\text { folhas } 100 \mathrm{~g}\end{array}$ & 0 & 76 & 102 & 100 & 68 & 18 & 30 & 30 & 0 & 424 \\
\hline $\begin{array}{l}\text { Melissa officinalis } \\
\text { folhas } 100 \mathrm{~g}^{*}\end{array}$ & 60 & 37 & 36 & 37 & 62 & 13 & 0 & 10 & 0 & 255 \\
\hline $\begin{array}{l}\text { Mentha } s p \\
\text { folhas } 100 \mathrm{~g} *\end{array}$ & 0 & 0 & 53 & 11 & 25 & 14 & 0 & 20 & 0 & 123 \\
\hline $\begin{array}{l}\text { Mikania glomerata } \\
\text { folhas } 100 \mathrm{~g} *\end{array}$ & 30 & 0 & 48 & 0 & 0 & 0 & 0 & 0 & 0 & 78 \\
\hline $\begin{array}{l}\text { Mikania glomerata } \\
\text { tintura } 20 \% 100 \mathrm{~mL}^{*}\end{array}$ & 10 & 24 & 21 & 30 & 65 & 20 & 19 & 0 & 0 & 189 \\
\hline $\begin{array}{l}\text { Passiflora alata } \\
\text { folhas } 100 \mathrm{~g}\end{array}$ & 24 & 0 & 37 & 47 & 16 & 40 & 0 & 0 & 0 & 164 \\
\hline $\begin{array}{l}\text { Passiflora alata } \\
\text { tintura } 10 \% 100 \mathrm{~mL}\end{array}$ & 39 & 40 & 24 & 80 & 44 & 40 & 15 & 19 & 20 & 321 \\
\hline $\begin{array}{l}\text { Persea gratissima } \\
\text { tintura } 20 \% 100 \mathrm{~mL}\end{array}$ & 32 & 24 & 11 & 20 & 40 & 20 & 12 & 10 & & 169 \\
\hline $\begin{array}{l}\text { Phyllanthus niruri } \\
\text { partes aéreas } 100 \mathrm{~g}\end{array}$ & 0 & 20 & 0 & 21 & 27 & 10 & 0 & 18 & 10 & 106 \\
\hline $\begin{array}{l}\text { Salvia officinalis } \\
\text { folhas } 100 \mathrm{~g}\end{array}$ & 0 & 20 & 10 & 35 & 37 & 0 & 0 & 15 & 27 & 144 \\
\hline
\end{tabular}


Tabela 2: Número de unidades farmacêuticas contendo espécies medicinais produzidas no Laboratório de Manipulação Farmacêutica (2003-2017)

\begin{tabular}{ccc}
\hline $\begin{array}{c}\text { Produto } \\
\text { manipulado } \\
\text { / } \\
\text { Ano }\end{array}$ & $\begin{array}{c}\text { Calendula } \\
\text { officinalis } \\
\text { pomada } \\
\mathbf{1 0 \% ~ 2 0 0 g}\end{array}$ & $\begin{array}{c}\text { Stryphnodendron } \\
\text { adstringens } \\
\text { creme 10\% } \\
\mathbf{2 0 0 g}\end{array}$ \\
\hline 2003 & 339 & 0 \\
2004 & 669 & 0 \\
2005 & 768 & 0 \\
2006 & 1033 & 0 \\
2007 & 1001 & 0 \\
2008 & 1594 & 0 \\
2009 & 2138 & 0 \\
2010 & 2618 & 0 \\
2011 & 2160 & 0 \\
2012 & 3022 & 0 \\
2013 & 3492 & 0 \\
2014 & 3770 & 155 \\
2015 & 3706 & 695 \\
2016 & 2347 & 614 \\
2017 & 385 & 68 \\
Total & 29779 & 1532 \\
\hline
\end{tabular}

Na tabela 1, observa-se a produção realizada entre os anos de 1994 a 2002, onde é apresentado o desempenho na primeira fase do Programa de Fitoterapia e Homeopatia, na qual os atendimentos foram somente realizados no Núcleo de Gestão Assistencial, possuindo um único clínico especialista para esta especialidade.

Observa-se que nessa fase houve predominância da produção de drogas vegetais e derivados vegetais (extratos vegetais na forma de tinturas na proporção 1:5). O elenco de espécies medicinais utilizado no desenvolvimento do programa foi idealizado observando-se a prevalência das doenças no município, em concordância com o uso de espécies medicinais, cujas alegações eram subsidiadas por estudos científicos. Nessa condição, havia o cultivo de algumas espécies medicinais no Horto Municipal de Ribeirão Preto (Cymbopogon citratus, Melissa officinalis, Mentha sp e Mikania glomerata), as quais eram transformadas em droga vegetal e tinturas em procedimentos realizados no Laboratório de Manipulação Farmacêutica.

Existia, também, a possibilidade de aquisição desses insumos em empresas especializadas nesses produtos, por via licitatória. Esses materiais eram recebidos no Laboratório de Manipulação Farmacêutica e, após procedimentos de controle de qualidade (identificação botânica, avaliação da pureza e análise qualitativa da composição química, usando cromatografia em camada delgada), eram fracionados em embalagens adequadas para dispensação ao paciente. Somente dois fitoterápicos foram produzidos nessa fase (alho - Allium sativum 500mg em cápsulas, calêndula Calendula officinalis $10 \%$ na forma farmacêutica de pomada).

Ressalta-se que, durante este período, o Programa de Fitoterapia e Homeopatia, 
isoladamente, ou em conjunto com a Direção Regional de Saúde de Ribeirão Preto, desenvolveram ações de capacitação junto aos profissionais da área da saúde interessados na terapêutica. Houve, em 1994, o curso de Treinamento em Fitoterapia; em 2001, o Fórum de Plantas Medicinais e, em 2002, o curso de Fitoterapia na Atenção Básica - SUS.

Nessa formatação do Programa de Fitoterapia e Homeopatia, houve a produção de 4.318 unidades de produtos à base de plantas medicinais, em nove anos de trabalho. Os produtos de maior demanda foram calêndula - Calendula officinalis pomada $200 \mathrm{~g}$ (com indicações para inflamações, lesões, contusões e queimaduras), espinheira santa Maytenus ilicifolia folhas $100 \mathrm{~g}$ (com indicação para dispepsia, azia, gastrite e coadjuvante no tratamento e prevenção de úlcera), maracujá - Passiflora alata tintura a $20 \% 100 \mathrm{~mL}$ (com indicação em quadros leves de ansiedade, insônia e como calmante suave) e alho - Allium sativum cápsulas $500 \mathrm{mg}$ (contendo extrato seco, indicado em quadros de hipercolesterolemia).

$\mathrm{Na}$ tabela 2, observa-se a produção nos anos entre 2003 a 2017, quando se desenvolveu a segunda fase do Programa de Fitoterapia e Homeopatia. Nesse momento, por fatores econômicos e políticos, as atividades relacionadas à Fitoterapia no Núcleo de Gestão Assistencial foram encerradas. O clínico fitoterapeuta se aposentou e não houve interesse dos gestores municipais na reposição do profissional. A partir daí, a demanda de preparações a base de espécies vegetais na rede municipal de saúde de Ribeirão Preto sofreu alterações.

Houve drástica redução da variedade de itens produzidos (de 21 itens passou para somente 1, até 2013 e depois para 2, até os dias atuais). No entanto, observou-se que a quantidade produzida desses itens aumentou de forma intensa até 2015, pois o uso desses fitoterápicos passou a ser adotado em todas as unidades da rede pública de saúde do município, tendo acesso para indicação desses produtos equipes multiprofissionais de saúde, com uso em salas de curativo, em serviço de atendimento domiciliar (SAD) e para uso do próprio paciente. Nessa condição, os fitoterápicos, apesar de ainda serem produzidos pelo Laboratório de Manipulação Farmacêutica, seus insumos, incluindo os derivados vegetais (tinturas), eram adquiridos por via licitatória, devido aos grandes volumes necessários para produção.

Desse modo, nessa fase, a calêndula - Calendula officinalis pomada, apresentada em embalagens de $200 \mathrm{~g}$, teve produção de quase 30.000 potes, totalizando 6,96 toneladas de pomada em 15 anos de produção. O creme de barbatimão - Stryphnodendron adstringens (indicado para lesões, como cicatrizante e antisséptico tópico na pele), produzido como descrito no Formulário de Fitoterápicos da Farmacopeia Brasileira (22), foi introduzido para uso em 2014. Essa inclusão foi efetivada a partir de demanda dos profissionais das salas de curativos e do $\mathrm{SAD}$, que desejavam uma alternativa à utilização da pomada de calêndula. Até 2017, uma quantidade superior a $300 \mathrm{Kg}$ desse creme foi produzida e utilizada no SUS de Ribeirão Preto.

Ao analisar todos os dados (tabelas 1 e 2), constata-se que o Laboratório de Manipulação Farmacêutica produziu 23 tipos de produtos, tendo como base espécies medicinais em sua composição, totalizando 35.629 unidades produzidas nesses 24 anos de atuação.

Em uma análise da evolução do número de unidades produzidas por ano (Figura 1), observa-se com nitidez as fases de atuação do Programa de Fitoterapia e Homeopatia que foram citadas anteriormente. A primeira fase, de 1994 a 2002, com diversidade de preparações e um único clínico com direito a prescrição e a segunda fase, de 2003 a 2015, com drástica diminuição da diversidade de preparações, mas um incremento no número de unidades produzidas e com a possibilidade de indicação de uso realizada por 
equipes multiprofissionais.

Essa situação só pôde ser constantemente ampliada com o passar dos anos, a partir de aquisição de novos equipamentos (com fomento obtido a partir de projeto de pesquisa junto à FAPESP, em 2006) e disponibilização de mais funcionários para o Laboratório de Manipulação Farmacêutica. Não obstante, nesse período, houve tentativas de obter financiamento para o programa e o laboratório, através da "Seleção de Propostas de Arranjos Produtivos Locais no Âmbito do SUS", junto à Secretaria de Ciência, Tecnologia e Insumos Estratégicos do Ministério da Saúde, em 2012 e 2013, sem, no entanto, obter êxito nessas incursões.

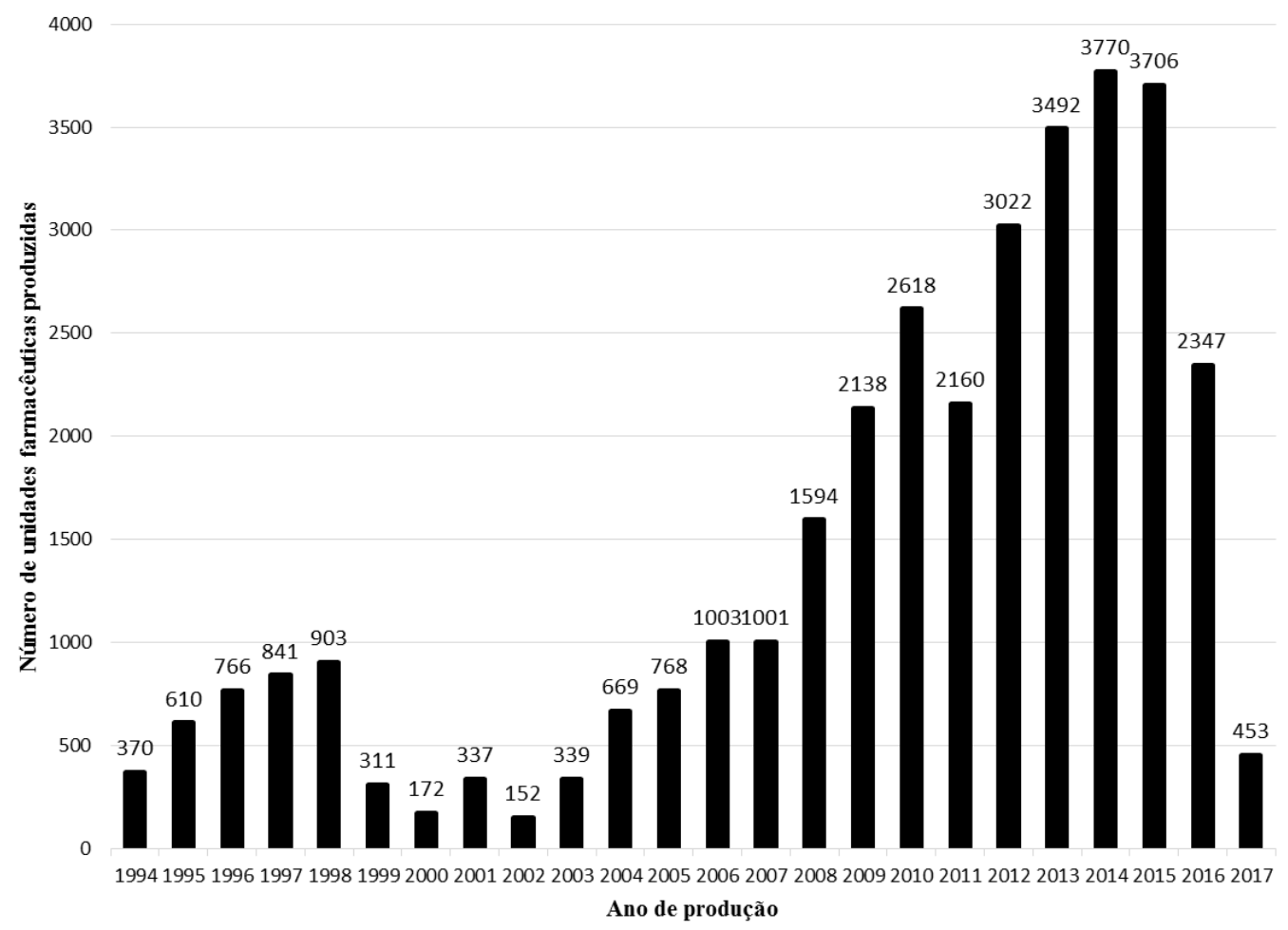

Figura 1: Número de unidades farmacêuticas contendo espécies medicinais produzidas no Laboratório de Manipulação Farmacêutica (1994-2017).

Realizando-se avaliação comparativa do histórico percorrido pelo Programa de Fitoterapia e Homeopatia na cidade de Ribeirão Preto, com o que foi discutido por Ribeiro (4), percebe-se certa analogia em relação aos acontecimentos descritos. Segundo esse autor, programas desse tipo existentes no Brasil tiveram um comportamento que, incialmente $(1980$ - 2008), contemplavam ações mais horizontais (envolvendo utilização de hortos públicos, ações com a comunidade, manipulação de fitoterápicos em estabelecimentos próprios) e que, dessa forma, apresentavam diversidades regionais. A partir de 2008 em diante, com o lançamento do Programa Nacional de Plantas Medicinais e Fitoterápicos no âmbito do SUS, paradoxalmente aos objetivos dessa política pública, os programas apresentaram uma transição, acomodando ações verticalizadas na escala nacional, perdendo as características regionais e usando, basicamente, fitoterápicos industrializados (produção em escala).

Ainda observando a Figura 1, nota-se que, em 2016 e 2017, houve forte desaceleração na produção desses produtos. Essa situação é consequência do momento caótico que alguns municípios e estados estão passando em relação à grave crise econômica, associada à crise política que tangencia, também em Ribeirão Preto, os meios policiais. Espera-se que, nos próximos anos, essa situação seja ultrapassada e a produção e 
distribuição de fitoterápicos seja normalizada em toda rede SUS do município.

Por outro lado, na contramão do que foi citado, a partir de junho de 2016, uma nova tentativa de instituir a Homeopatia no município se iniciou, com a prescrição sendo realizada por vários médicos especialistas da rede pública de saúde e os medicamentos sendo produzidos pelo Laboratório de Homeopatia da Secretaria da Saúde de Ribeirão Preto (anexo ao Laboratório de Manipulação Farmacêutica), com a inclusão de um farmacêutico homeopata, com a responsabilidade de produção e controle desses medicamentos.

Ao mesmo tempo, o programa desenvolvido, atualmente, em Ribeirão Preto, intitulado "Programa Municipal de Práticas Integrativas e Complementares em Saúde", apresentou aos novos gestores municipais, no início de 2017, um projeto que desenha a implantação de um núcleo de atendimento nessa área, que envolverá, no mesmo local, a disponibilização à população de vários tipos de terapêuticas descritas no âmbito da Política Nacional de Práticas Integrativas e Complementares, além de área para cultivo de espécies medicinais, manipulação e produção de produtos homeopáticos e fitoterápicos.

No final de 2017, proposta para desenvolvimento de ações de extensão, tendo como foco a Fitoterapia e a estruturação de uma Farmácia de Manipulação de Fitoterápicos no município de Ribeirão Preto, foi envidada à Secretaria de Ciência, Tecnologia e Insumos Estratégicos do Ministério da Saúde (Chamada Pública SCTIE/MS no 1/2017). Entre dezenas de propostas, a de Ribeirão Preto foi uma das selecionadas. Dessa forma, acreditamos que um novo ciclo, com boas perspectivas para o desenvolvimento do Programa e do Laboratório se inicia a partir desse financiamento federal.

\section{Considerações finais}

Após análise do desempenho em relação à produção de drogas vegetais, derivados vegetais e fitoterápicos pelo Laboratório de Manipulação Farmacêutica para o Programa de Fitoterapia e Homeopatia da Secretaria da Saúde de Ribeirão Preto, no período de 1994 a 2017, foi observado que:

Grande força em relação à manutenção das atividades durante esse período esteve ligada à necessidade que a população demonstrou em ter viáveis no serviço público esse tipo de tratamento e medicamento.

Há necessidade de usar os meios e recursos disponíveis para que esses serviços se concretizem e sejam permanentes. De forma oportuna, houve solicitação de recursos às instituições de fomento para esses serviços e desenvolvimento de ações cooperativas com instituições de Ensino Superior.

Deve-se observar a qualidade desses serviços junto à população. Isso envolveu o atendimento ao paciente e o medicamento disponibilizado. Almejou-se trabalhar para se obter grau de excelência na execução dessas ações. Caso não seja dessa forma, há possibilidade de não se obter o reconhecimento e apoio da população.

Existiram e ainda persistem variados fatores que interferem na perenidade e no desenvolvimento contínuo de programas desse tipo, que vão desde aspectos ideológicos, políticos e econômicos, que podem envolver o poder executivo e legislativo em nível municipal, estadual e federal, bem como os gestores da área da saúde.

Fonte de financiamento: FAPESP processo no 2006/64408-3 


\section{Vittalle - Revista de Ciências da Saúde v. 30, n. 1 (2018) 46-55}

\section{Referências}

1. Brasil. Ministério da Saúde. Secretaria de Políticas de Saúde. Projeto Promoção da Saúde. As Cartas da Promoção da Saúde. Brasília: Ministério da Saúde; 2002.

2. Matsuchita HLP, Matsuchita ASP. A Contextualização da Fitoterapia na Saúde Pública. Uniciências 2015; 19 (1):86-92.

3. Brasil. Ministério da Saúde. Secretaria de Atenção à Saúde. Departamento de Atenção Básica. Práticas integrativas e complementares: plantas medicinais e fitoterapia na atenção básica. Brasília: Ministério da Saúde; 2012.

4. Santos RL, Guimaraes GP, Nobre MSDC, Portela ADS. Análise sobre a fitoterapia como prática integrativa no Sistema Único de Saúde. Revista Brasileira de Plantas Medicinais 2011; 13(4): 486-91.

5. Fernandes TM. Plantas medicinais: memória da ciência no Brasil. Rio de Janeiro: Editora Fiocruz, 2004.

6. Figueredo CA, Gurge IGD, Junior GDG. A Política Nacional de Plantas Medicinais e Fitoterápicos: construção, perspectivas e desafios. Physis-Revista de Saúde Coletiva 2014; 24(2): 381-400.

7. Brasil. Ministério da Saúde. Secretaria de Atenção à Saúde. Departamento de Atenção Básica. Política Nacional de Práticas Integrativas e Complementares no SUS. Brasília: Ministério da Saúde; 2006.

8. Brasil. Ministério da Saúde. Secretaria de Ciência, Tecnologia e Insumos Estratégicos. Departamento de Assistência Farmacêutica. Política Nacional de Plantas Medicinais e Fitoterápicos. Brasília: Ministério da Saúde; 2006.

9. Ribeiro LH. Análise dos programas de plantas medicinais e fitoterápicos no Sistema Único de Saúde (SUS) sob a perspectiva territorial [Internet]. Ciência \& Saúde Coletiva; 2017 - [citado em 2017 out 25]. Disponível em: http://www.cienciaesaudecoletiva.com.br/artigos/analise-dos-programas-deplantas-medicinais-e-fitoterapicos-no-sistema-unico-de-saude-sus-sob-a-perspectivaterritorial/16382? $\mathrm{id}=16382$

10. Ribeirão Preto. Secretaria Municipal da Saúde. Relatório anual de gestão: 2014 [Internet]. 2014 [citado em 2017 out 22]. Disponível em: https://www.ribeiraopreto.sp.gov.br/ssaude/pdf/2014relatorio_gestao.pdf

11. Pires AM, Borella JC, Raya LC. Prática alternativa de saúde na atenção básica da rede SUS de Ribeirão Preto (SP). Divulgação em Saúde para Debate 2004; 30:56-58.

12. Antonio GD, Tesser CD, Moretti-Pires RO. Contribuições das plantas medicinais para o cuidado e a promoção da saúde na atenção primária. Interface-Comunicação, Saúde, Educação 2013; 17(46): 615-633.

13. Borella JC, Pereira LHTR. Produção e avaliação comparativa de preços de produtos do Laboratório de Manipulação Farmacêutica - Ribeirão Preto - SP: experiência relacionada à assistência farmacêutica no SUS. Cadernos de Saúde Coletiva 2017; 25(2): 210-216.

14. Borella JC, Ribeiro NS, Teixeira JCL, Carvalho DMA. Avaliação da espalhabilidade e do teor de flavonoides em forma farmacêutica semissólida contendo extratos de Calendula officinalis L. (Asteraceae). Revista de Ciências Farmacêuticas Básica e Aplicada 2010; 31(2): 193-197.

15. Borella JC, Ribeiro NS, Freato AMR, Mazzo KF, Barbosa DM. Influência da adubação e da cobertura morta na produtividade e no teor de flavonóides de Calendula officinalis L. (Asteraceae). Revista Brasileira de Plantas Medicinais 2011; 13(2): 235-239.

16. Borella JC, Carvalho DMA. Avaliação comparativa da qualidade de extratos de Calendula officinalis L. (Asteraceae) comercializados em farmácias de manipulação em Ribeirão Preto - SP. Revista Brasileira de Farmácia 2011; 92(1): 11-16.

17. Borella JC, Ribeiro NS, Teixeira JCL, Carvalho DMA. Influência do processo extrativo nas propriedades físico-químicas dos extratos de Calendula officinalis L. (Asteraceae). Revista Eletrônica de Farmácia 2012; 9(2): 25-36.

18. Borella JC, Teixeira JCL. Avaliação comparativa de certificados de análises de empresas que comercializam tintura de Calendula officinalis L. (Asteraceae). Visão Acadêmica 2013; 14(3): 26-35.

19. Fernandes EFA, Meloni F, Borella JC, Lopes NP. Effect of fertilisation and harvest period on polar metabolites of Calendula oficcinalis. Revista Brasileira de Farmacognosia 2013; 23:731-735. 
20. Borella JC, Marincek A, Rodrigues MF. Avaliação comparativa da composição química de drogas vegetais e extratos de Calendula officinalis L. (Asteraceae) cultivada com variação na adubação e na cobertura de solo. Visão Acadêmica 2014; 15(1): 15-26.

21. Brasil. Ministério da Saúde. Agência Nacional de Vigilância Sanitária. Memento Fitoterápico da Farmacopeia Brasileira. 1ª . ed. Brasília: Agência Nacional de Vigilância Sanitária; 2016.

22. Brasil. Ministério da Saúde. Agência Nacional de Vigilância Sanitária. Formulário de Fitoterápicos da Farmacopeia Brasileira. 1ª . ed. Brasília: Agência Nacional de Vigilância Sanitária; 2011. 